\title{
The Efficacy of Daclizumab for Intestinal Transplantation: Preliminary Report
}

\author{
K. Abu-Elmagd, J. Fung, W. McGhee, D. Martin, G. Mazariegos, N. Schaefer, J. Demetris, T.E. Starzl, \\ and J. Reyes
}

$\mathrm{W}$ E PREVIOUSLY identified rejection as the most significant risk factor for intestinal allograft survival. ${ }^{1}$ The reported efficacy of daclizumab (Zenapax. Hoffman-LaRoche) prophylaxis with solid organ transplantation $^{2}$ triggered its use at our center as induction therapy for intestinal transplantation.

\section{MATERIALS AND METHODS}

Daclizumab was used as prophylactic therapy for a total of 14 consecutive patients who received primary intestinal transplantation under tacrolimus-based immunosuppression during the period from May 31, 1998 to March 31, 1999. Of these, eight (57\%) were isolated intestine, and six (43\%) were liver/intestine. Eight were children and seven were female patients. Daclizumab was given intravenously in a dose of 1 to $2 \mathrm{mg} / \mathrm{kg}$ body weight for a total of five doses. The first dose was administered within a few hours before surgery and the remaining four doses were given postoperatively at $2,4,6$, and 8 weeks after transplantation. In only one recipient (isolated intestine), the drug was discontinued after the second dose because of unexplained coagulopathy and recurrent gastric bleeding. Cellcept or imuran were given from the outset in nine recipients.

All donors were cadaveric and ABO identical, and HLA matching was random.

No attempts were made to immunomodulate any of the grafts with antilymphoid antibody treatment, irradiation, or other modalities, and six patients received donor bone marrow augmentation. The crossmatch was positive with dithiothreitol (DTT) in four cases (two intestine. two liver/intestine). The mean (SD) cold ischemia time was $9.7 \pm 2.7$ hours. Details of the donor/recipient operations, and bone marrow augmentation technique have been described elsewhere. ${ }^{3-5}$ The observation period was limited to the first postoperative year with August 30, 1999 being the date of last follow-up.

Acute rejection was diagnosed by histopathologic examination of random and endoscopically guided multiple mucosal biopsies. The adopted diagnostic criteria have been described elsewhere. ${ }^{\circ}$ A new rejection episode was defined by documentation of new histologic changes. All of the rejection episodes were histologically documented and medically treated. OKT3 was used to treat steroidresistant rejection.

\section{RESULTS}

With a mean follow-up of $8.5 \pm 3.9$ months, $11(79 \%)$ recipients were alive with fully functioning grafts. The three deaths (two liver/intestine, one isolated intestine) occurred at 4, 56, and 225 days after transplantation. The causes were primary graft failure, rejection, and renal failure. The primary failed liver/intestinal graft was given to a black pediatric recipient across a strong positive crossmatch (titer: 1:512). The 1-year Kaplan-Meier TPN-free survival rate was $77 \%$. Daclizumab prophylaxis resulted in a significant reduction in the incidence of rejection during the study period. With a mean follow-up of $8.5 \pm 3.9$ months, only six $(43 \%)$ recipients experienced at least one episode of acute rejection at a mean onset of $22 \pm 18$ days after transplantation. The incidence of rejection was $30 \%$ at 1 month, $55.6 \%$ at 3 months, and $43 \%$ at 6 months, with a mean number of episodes/patient of $0.4 \pm 0.7,1.0 \pm 1.1$, and $1.1 \pm 1.8$, respectively. OKT3 was used to treat steroid-resistant rejection in four (29\%) recipients.

CMV was reactivated in one $(7 \%)$ recipient at 121 days after transplantation, and PTLD was diagnosed in another (7\%) patient during postoperative week 7 . Both cases were treated successfully with antiviral therapy and careful reduction in immunosuppression.

The mean total hospital stay was $4.6 \pm 2.2$ weeks with an ICU stay of $7.6 \pm 6.5$ days. Graft function was restored and TPN was discontinued at a median time of 20 days (range 8 to 35) from date of transplantation.

\section{DISCUSSION}

These preliminary data show that daclizumab is beneficial as an induction therapy for intestinal allograft recipients. The risk of rejection during the first 6 months was significantly reduced with a relatively low incidence of opportunistic infections and short initial hospital stay. ${ }^{1.4}$ In conclusion, daclizumab prophylaxis is an effective therapy for

From the Thomas E. Starzl Transplantation Institute, University of Pittsburgh Medical Center, Pittsburgh, Pennsylvania, USA.

Address reprint requests to $\mathrm{Dr}$ Kareem Abu-Elmagd, Thomas E. Starzl Transplantation Institute, Fourth Floor Falk Clinic, 3601 Fifth Avenue, Pittsburgh, PA 15213. E-mail: kae@med.pitt.edu. 
intestinal transplantation. However, its long-term therapeutic and survival benefits have yet to be defined.

\section{REFERENCES}

1. Abu-Elmagd K, Reyes J. Todo S. et al: J Am Coll Surg 186:512. 1998

2. Vincenti F, Kirkman R. Light S. et al: N Engl J Med 338:161, 1998
3. Starzl TE, Todo S, Tzakis A, et al: Surg Gynecol Obstet 172:335, 1991

4. Abu-Elmagd K. Todo S. Tzakis A. et al: Surg Gynecol Obstet 179:385. 1994

5. Fontes P, Rao A, Demetris AJ, et al: Lancet 344:151, 1994

6. Lee RG. Nakamura K. Tsamandas AC. et al: Gastroenterology 110:1820, 1996 